\title{
Remote Sensing Observations of Dominant Breaking Waves in Intermediate to Deep Water from a Lighthouse During Storm Conditions
}

\author{
Caio Eadi Stringari ${ }^{1, *} \quad$, Jean-François Filipot ${ }^{1}{ }^{\text {, Fabien Leckler }}{ }^{1}$, Rui Duarte ${ }^{1}$ \\ 1 France Energies Marines, 25 Avenue Alexis de Rochon, 29280 Plouzané, France \\ * Correspondence: Caio.Stringari@France-Energies-Marines.org; \\ Jean-Francois.Filipot@France-Energies-Marines.org
}

\begin{abstract}
Wave breaking is one of the most important yet poorly understood water wave phenomena. It is via wave breaking that waves dissipate most of their energy and the occurrence of wave breaking directly influences several environmental processes, from ocean-atmosphere gas exchanges to beach morphodynamics. Large breaking waves also represent a major threat for navigation and for the survivability of offshore structures. This paper provides a systematic search for intermediate to deep water breaking waves with particular focus on the elusive occurrence of plunging breakers. Using modern remote sensing and deep learning techniques, we identify and track the evolution of over four thousand unique wave breaking events using video data collected from La Jument lighthouse during ten North Atlantic winter storms. Out of all identified breaking waves $\left(N_{b}=4683\right), \approx 22 \%$ were dominant breaking waves, that is, waves that have speeds within $\left[0.77 c_{p}, 1.43 c_{p}\right]$, where $c_{p}$ is the peak wave speed. Correlations between the occurrence rate of dominant breaking waves (that is, waves per area and time per peak wave period) and wave steepness and wave age were observed. As expected, the number of identified plunging waves was small and six waves of all detected breaking waves, or $0.13 \%$, could undoubtedly be considered as plunging waves. Two waves were also identified as unusually large, or rogue waves. Although afflicted by several technical issues, the data presented here provides a good indication that the probability of occurrence of plunging waves should be better incorporated into the design of offshore structures, particularly the ones that aim to harvest energy in offshore environments.
\end{abstract}

Keywords: wave breaking; remote sensing; intermediate water waves, dominant waves; plunging waves; rogue waves; machine learning. 


\section{Introduction}

Wave breaking is still one of the least understood water wave phenomena despite decades of continuous research, especially during extreme storm conditions. LonguetHiggins [1] summarizes the occurrence of large scale intermediate to deep water wave breaking into two main categories: spilling and plunging breakers. In a spilling breaker, a region of strong turbulence, visible as white foam, develops on the crest of the wave as it propagates. Plunging breakers are far more dramatic as the wave profile becomes so unstable that the wave crest curls and a forward moving (plunging) jet is formed. Breaking waves in intermediate to deep water result from wave group interactions [2] or may be generated by the hydrodynamic modulation of short waves by underlying long waves, which increases the probability of wave breaking of the short waves [3]. While the occurrence of spilling breakers is easily observed during moderate to strong wind conditions, direct field observations of intermediate to deep water plunging breakers remains rare and, to the authors' knowledge, only laboratory data [4-6] or reports from mariners [7] are available. Further, contrarily to their shallow water counterparts, no non-dimensional number to differentiate between the two types of breakers is currently available in the literature, which makes their identification very challenging using conventional instruments such as wave buoys or pressure transducers.

This paper will focus on the occurrence of dominant breaking waves, which are defined as waves that have energy contained within $\pm 30 \%$ of the spectral peak frequency $\left(f_{p}\right)$ and are assumed to correspond to the peak enhancement region in the JONSWAP spectrum [2]. Assuming that the linear dispersion relation is valid, dominant waves are, consequently, waves that travel at speeds close to the peak spectral speed $\left(c_{p}\right)$, within the range $\left[0.77 c_{p}, 1.43 c_{p}\right]$ (not accounting for bottom interaction). Despite this definition being rather arbitrary, it is practical and supported by the fact that the most intense and dangerous breaking waves are the dominant waves, since they carry most of the energy of the wave field. The research presented here will, therefore, heavily rely on such definition.

The main candidates for intermediate to deep water dominant breakers in well developed, storm seas may be the unusually large waves known was rogue waves. For example, a laboratory investigation of the Draupner wave has recently shown that plunging breaking happened for one of the possible simulated scenarios [5]. There also is some debate regarding if another famous rogue wave, the Andrea wave, was a breaking wave or not [8] (the evidence points towards no). In contrast, the rogue 
wave recently reported by Cavaleri et al. [9] was evidently breaking (most probably as a strong spilling breaker). The mechanisms that generate rogue waves are also not fully understood. While the Benjamin-Feir (or modulational) instability has been used to justify such waves (see Adcock and Taylor [10] for a review), recent research shows that it is not a requirement [11]. Further, interactions between the waves and the underlying current field [12] also enhance the probability of occurrence of rogue waves and the interaction with strong opposing winds may considerably steepen breaking waves, at least in shallow water [13]. All the mentioned factors, as well as direct observation of unusually large waves [14], occur in the vicinity of La Jument lighthouse, which makes this location a perfect spot for the search of large breaking waves.

In the context of the current climate crisis and the search for renewable offshore energies, it is important to make a clear distinction between the occurrence of plunging and spilling breakers in the design of offshore structures given that the forces that these waves generate on structures are very distinct. While spilling breakers generate continuous forces, the forces from plunging breakers may be felt as sharp peaks [15], known as "slamming forces". Considering the famous Goda formula [16], the slamming force of a breaking wave is:

$$
F_{I}=\rho_{w} D \lambda \eta_{b} c_{b}^{2} C_{s}
$$

where $\rho_{w}$ is the water density, $D$ is the structure diameter, $c_{b}$ is the breaking wave speed, $\eta_{b}$ is the maximum water elevation above the still water level, $C_{S}$ is a constant (equals to $\pi$ in Goda's work [16]) and $\lambda$ is a wave curling factor which gives the proportion of the crest that is active in the slamming load. Assuming that only plunging waves have non-negligible $\lambda$, knowing the probability of occurrence of such waves is of crucial importance for the design of offshore structures such as floating wind turbines.

In this paper, we employ state-of-the-art remote sensing and image processing techniques applied to a dataset of extreme waves collected at La Jument lighthouse off the coast of Brittany, France during the 2019-2020 North Atlantic winter storm season (ten storms are analyzed here). Our efforts aim to provide further insight into dominant, intermediate to deep water wave breaking with particular focus on the occurrence of plunging breakers, as these waves represent a major threat for ships and offshore structures. The La Jument lighthouse is the ideal location for observing extreme dominant breaking waves in deep and intermediate water using remote sensing instrumentation. This iconic lighthouse acts as a real-world laboratory providing a high 
vantage point on a steep shelf-break that can be easily accessed and equipped during the summer. Further, it is fully exposed to North Atlantic storm waves (individual wave height recorded over $24 \mathrm{~m}$ by Filipot et al. [14]) and its location is close to future commercial floating offshore wind farms to be deployed in Brittany. Our results indicate that, from a total number of over four thousand active wave breaking events, $21.7 \%$ were classified as dominant waves and six waves could be undoubtedly considered plunging breakers. The occurrence rate of breaking waves correlated well with wave steepness and wave age. This paper is organized as follows: Section 2.1 presents a summary of the field observations, Section 2.2 describes how breaking waves were detected, Sections 3 and 4 present, respectively, results and discussions regarding dominant breaking waves and the occurrence of plunging waves and Section 5 concludes.

\section{Materials and Methods}

\subsection{Field Observations}

Field observations were conducted during the 2019-2020 North Atlantic winter storm season and covered a span of three months (from mid October 2019 to mid January 2020). Stereo video data were collected at the La Jument lighthouse off the coast of Brittany, France $\left(48^{0} 25^{\prime} 20.00^{\prime \prime} \mathrm{N}, 5^{0} 8^{\prime} 2.28^{\prime \prime} \mathrm{W}\right)$ using the same instruments described in Filipot et al. [14]. The data used in this paper were collected by a pair of synchronized 5 megapixel (2048 x 2456 pixels) BM-500GE JAI cameras fitted with 64mm lenses. Differently from the previous study, in which the cameras imaged the area directly below the lighthouse, in the present study, the cameras imaged water depths from $60 \mathrm{~m}$ in the near field of view (about 200m away from the lighthouse) to $120 \mathrm{~m}$ in the far field of view (about $1 \mathrm{~km}$ away from the lighthouse). Assuming an average depth $(d)$ of $90 \mathrm{~m}$, $k d$ ranged from $\approx 1.2$ to $\approx 3.3$, with $k$ the wavenumber. Figure 1 and Table 1 show the environmental characteristics during the campaign. In addition to the stereo video cameras, a Datawell waverider buoy was deployed by the French Naval Hydrographic and Oceanographic Service (Shom) in the same area imaged by the video cameras (see Figure 2-a for the location of the buoy). A total of ninety 30-minute long video data acquisition runs were recorded but only ten 20-minute records, representative of storm conditions, will be analysed here, as it will be explained below. Note that the wave buoy data collection started after 1 month from the start of the campaign; therefore, the data reported here previous to this date was extracted from the HOMERE database $[17,18]$. The wind data reported here is from the European Center for Medium Range Weather Forecast ERA-5 reanalysis [19]. 
Extreme events were selected based on a block maxima approach [20] with a window size of three days (roughly corresponding to the storm passage rate in the region) applied to the significant wave height $\left(H_{m 0}\right)$ timeseries. A total of ten storms were identified using this method. Note, however, that not all the storms matched stereo video records (for example, the gap between November 12 to November 27). From the analysis of Table 1, the significant wave height for these storms varied from approximately $4 \mathrm{~m}$ up to $10 \mathrm{~m}$, the peak period varied from $10 \mathrm{~s}$ to $18 \mathrm{~s}$ and the peak wave direction ranged from SW to NW. The winds speeds varied from $11 \mathrm{~ms}^{-1}$ up to approximately $20 \mathrm{~ms}^{-1}$ with main direction also ranging from SW to NW. The computed wave ages $\left(c_{p} / u_{*}\right)$ varied from approximately 20 to 66 , which is an indicative of mature sea-states dominated by swells.

Table 1: Environmental conditions for the selected storm events. $H_{m 0}$ is the significant wave height, $T_{p}$ is the peak wave period, $S_{p}$ is the wave steepness calculated as $H_{m 0} / L$ in which $L=\frac{g}{2 \pi} T_{p}^{2}, u_{10}$ is the wind speed, $u_{d i r}$ is the wind direction, $c_{p} / u_{*}$ is the wave age and $D_{p}$ is the peak wave direction. All dates are in UTC time.

\begin{tabular}{lllllllll}
\hline Date & $\begin{array}{l}H_{m 0} \\
{[m]}\end{array}$ & $\begin{array}{l}T_{p} \\
{[s]}\end{array}$ & $\begin{array}{l}S_{p} \\
{[-]}\end{array}$ & $\begin{array}{l}u_{10} \\
{\left[m s^{-1}\right]}\end{array}$ & $\begin{array}{l}\left.u_{d i r}{ }^{o}\right] \\
c_{p} / u_{*} \\
{[-]}\end{array}$ & $\begin{array}{l}D_{p} \\
{\left[{ }^{o}\right]}\end{array}$ & $\begin{array}{l}k d \\
{[-]}\end{array}$ \\
\hline 2019-10-26 09:53:48 & 4.68 & 11.24 & 0.024 & 15.88 & 205 & 28.32 & 237 & 2.88 \\
2019-11-02 07:34:01 & 6.23 & 12.20 & 0.027 & 19.31 & 255 & 23.61 & 260 & 2.46 \\
2019-11-04 15:04:57 & 5.93 & 12.82 & 0.023 & 17.58 & 261 & 28.18 & 257 & 2.25 \\
2019-11-07 15:36:02 & 3.84 & 11.36 & 0.019 & 11.05 & 260 & 46.2 & 265 & 2.82 \\
2019-11-12 08:25:27 & 4.67 & 18.87 & 0.008 & 12.3 & 306 & 66.69 & 263 & 1.21 \\
2019-11-27 10:00:05 & 5.68 & 10.53 & 0.033 & 16.27 & 264 & 25.68 & 243 & 3.27 \\
2019-12-09 09:10:37 & 9.98 & 18.18 & 0.019 & 16.3 & 318 & 44.23 & 261 & 1.27 \\
2019-12-13 09:00:05 & 7.75 & 13.33 & 0.028 & 17.95 & 293 & 28.49 & 259 & 2.10 \\
2019-12-15 11:42:58 & 6.40 & 15.38 & 0.017 & 13.24 & 227 & 49.37 & 256 & 1.64 \\
2019-12-22 11:00:05 & 7.49 & 16.67 & 0.017 & 15.61 & 271 & 42.98 & 241 & 1.45 \\
\hline
\end{tabular}

Unfortunately, our experiments were afflicted by a series of technical issues due to the hostile environment surrounding the lighthouse which precluded from precisely obtaining individual wave parameters (for example, crest height and asymmetry). As a consequence, the analyses presented hereafter will have a more qualitatively character. Two weeks after the video recordings started, a short circuit in the camera housing cleaning wipers made them to work continuously. This means that, on average, the cleaning wiper blades contaminated approximately $70 \%$ of the images from the right camera. Further, strong winds caused the camera housing structure to vibrate significantly during the data acquisition runs. Because the cameras were imaging a significantly far field of view, even small vibrations may lead to differences of hundreds of meters in the far field. These two problems make the three-dimensional stereo reconstruction for the vast majority of the data either impossible or very unreliable. It was possible, nevertheless, 

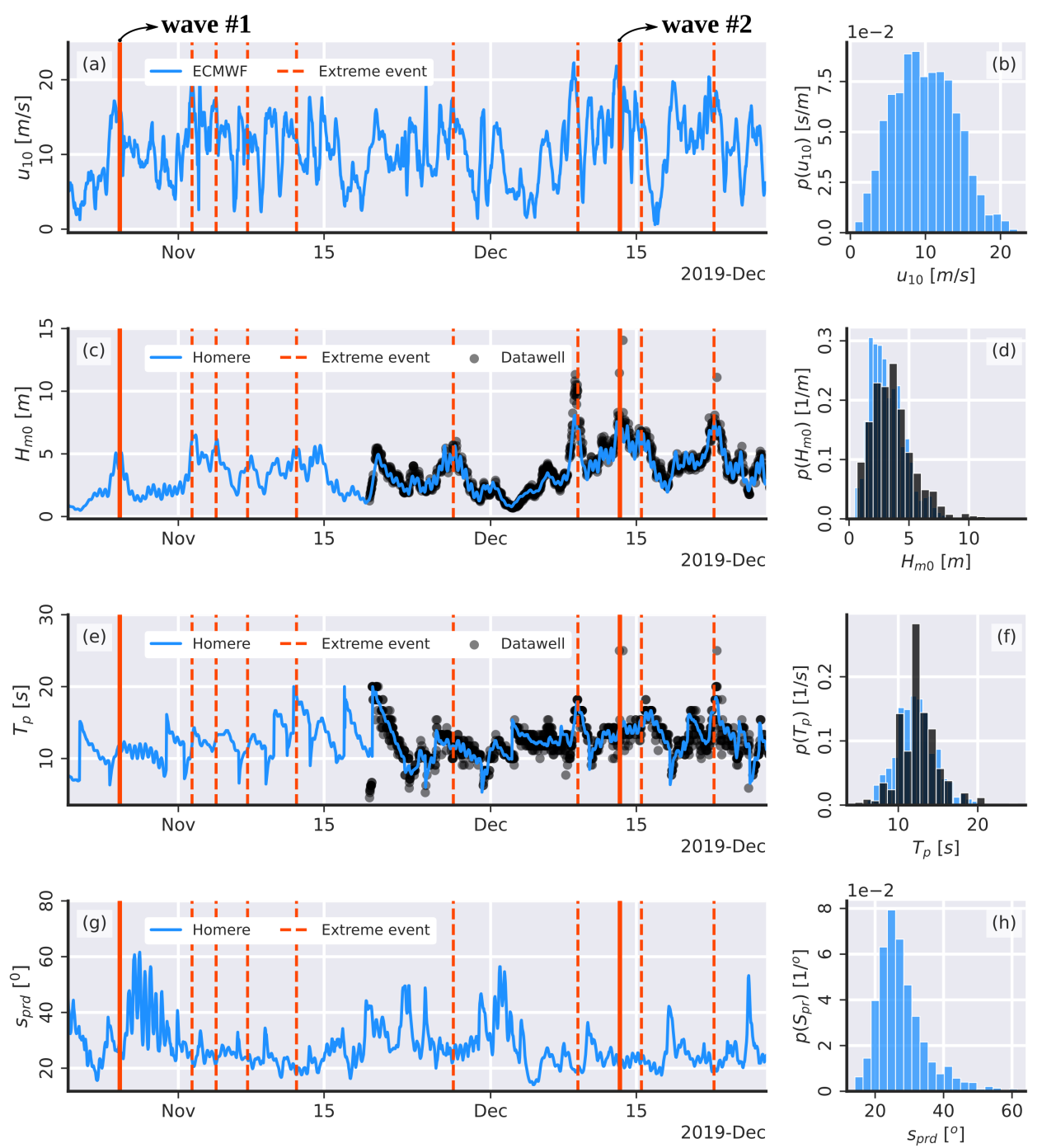

Figure 1. Wind and wave data during the video data acquisition period. (a) Timeseries of wind speeds at $10 \mathrm{~m}\left(u_{10}\right)$ from ECMWF's ERA5 reanalyses database [19]. (b) Histogram showing the probability density of $u_{10}$. (c) Timeseries of the significant wave height $\left(H_{m 0}\right)$ extracted from the HOMERE database $[17,18]$ (blue lines) and measured by the Datawell buoy (black circles). (d) Histogram showing the probability density of $H_{m 0}$ for both HOMERE (blue) and Datawell (black) data. (e) Timeseries of peak wave period $\left(T_{p}\right)$ extracted from the HOMERE database (blue) and measured by the Datawell buoy (black). (f) Histogram showing the probability density of $T_{p}$ for both HOMERE (blue) and Datawell (black) data. (g) Timeseries of directional spreading $\left(s_{\text {prd }}\right)$. (h) Histogram showing the probability density of $S_{p d}$ for HOMERE (blue). In (a), (c), (d) and (h), the vertical orange lines indicate the events classified as extreme and wave \#1 and \#2 indicate the conditions during the two waves discussed in Section 3.2. 
to use the standard ARGUS [21] methodology to project pixel to metric coordinates (that is, rectify the image) and utilise data from one of the cameras (the left camera) to track the evolution of breaking waves. To do so, 250 ground control points (GCPs) were extracted from a successful stereo reconstruction (from the day the cameras were installed and calibrated in calm conditions). A good agreement between measured and projected GCPS was found (Figures 2-a and b). Due to the characteristics of the field of view, the errors (both mean square error, RMSE, and mean absolute error, MAE) in the $x$-direction were only in the order of $0.1 \mathrm{~m}$ whereas the same errors in the $y$-direction are in the order of $1.5 \mathrm{~m}$. Based on this results, all usable images were projected to metric coordinates considering the tidal level as estimated by Boudière et al. [17] (which is provided alongside HOMERE data) as projection height.
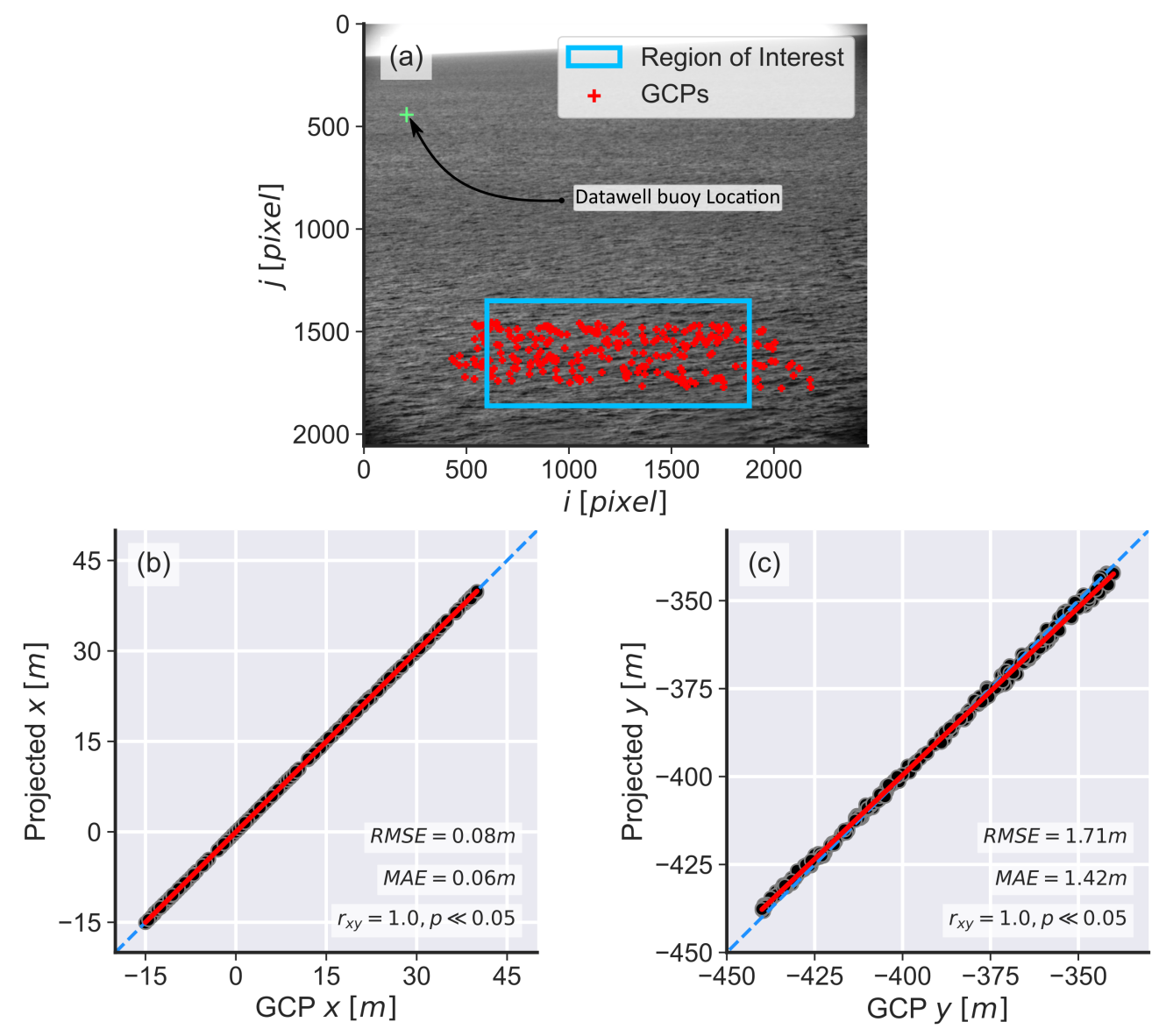

Figure 2. (a) Snapshot showing the region of interest and the points used as ground control points (GCPs) for image rectification. The blue rectangle shows the region of interest and has area $(A)$ equals to $8107.9 \mathrm{~m}^{2}$. (b) Comparison between measured and projected $\mathrm{x}$-coordinates. (c) Comparison between measured and projected y-coordinates. In (b) and (c), the red line shows the linear correlation between measured and projected coordinates, the blue dashed line shows the one-to-one equivalence line, RMSE is the root mean square error, $M A E$ is the mean absolute error and $r_{x y}$ is the Pearson's correlation coefficient. 


\subsection{Wave Breaking Detection}

The wave breaking detection technique used in this paper is an extension from the method developed in Stringari et al. [22]. These authors developed different neural networks trained on a relatively diverse dataset to classify images that contained active wave breaking, that is, breaking waves that are actively generating bubble entrainment. Differently from the original method, which is limited to a binary image classification task, here the technique is extended to an image segmentation framework. Such extension allows to classify individual pixels in an image as being active wave breaking or otherwise. A modified U-net model based on the Xception architecture $[23,24]$ was used as the deep learning backbone (Figure 3-a). A total of 2393 images divided into training $(80 \%)$ validation $(10 \%)$ and testing $(10 \%)$ datasets were manually annotated in a pixel-by-pixel manner and used to train and assess the neural network. The neural network weights from the best performing model in the previous study were used to initiate the present model (that is, transfer learning [25] was used). After the training procedure, the average classification error of the neural network is less than $5 \%$ for the training and validation datasets. However, errors as high as $25 \%$ for some samples in the test dataset were observed. Figure 3-b shows the evolution of the loss (or cost) function (binary cross-entropy) as a function of the training steps (or epochs) for the training and validation datasets and Figure 3-c shows the evolution of the accuracy score for the same datasets. Figure 3-d to $f$ show the application of the method to a sample drawn from the test dataset. These figures show how the neural network was capable to correctly classify the vast majority of the pixels but small errors, particularly for small scale breakers, remains. We encourage future researchers to attempted to further improve our method, which is fully open-source (see the Data Availability section for details).

To track the the spatio temporal evolution of the waves, a combination of data clustering and Kalman filtering was used. Individual active wave breaking pixels are clustered into patches are using the OPTICS clustering algorithm [26]. To be considered a correct detection, a minimum number of 10 pixels or maximum distance of 1 meter were required. The time evolution tracking of the actively breaking foam patches was done using the SORT [27] algorithm employing its default parameters. This method uses a combination of a Kalman filter and the Hungarian algorithm to track the time evolution of the bounding box defined from the clusters obtained with OPTICS. From the results of this algorithm, it is possible to obtain the speed at which the breaking wave crests are travelling $\left(c_{b}\right)$. Finally, wave breaking crest lengths were calculated 
using the $\alpha$-shape algorithm [28] (red lines in Figure 3-f) and ellipses were fitted to each detected event as per Stringari et al. [22]. While these data were not analysed in detail here, it allows, for example, to obtain Phillips' $\Lambda(c) d c$ distribution [29] from which wave breaking probabilities can be derived.

\section{Results}

\subsection{Wave Breaking Statistics}

Table 2 shows a summary of the number of observed breaking waves. A total of 4683 unique wave breaking events were identified across the ten storms. Of these, a total of 1121 detections (or $21.7 \%$ ) fell within the dominant wave breaking band as defined as per Banner et al. [2]. As expected, only a small number of breaking waves could be considered plunging waves. Six waves (or $0.13 \%$ ) of all detected breaking waves could undoubtedly be considered as plunging waves (which is equivalent to $0.53 \%$ of all dominant breaking waves). Note that all plunging waves were also dominant waves. To be considered a plunging wave, the wave must had presented the characteristic shape of a plunging wave and trapped air must had been observed. Unfortunately, more precise definitions of plunging waves, such as the one from Grilli et al. [30], could not be used due to the lack of three-dimensional data. See Section 3.2 below for more details about these waves.

Our analyses start with the kinematic and geometric properties of breaking waves. Figure 4-a shows the probability density distribution (PDF) and Kernel Distribution Function (KDE) of the ratio between wave breaking and peak phase speed $\left(p\left(c_{b} / c_{p}\right)\right)$ for all breaking waves. This PDF followed a log-normal distribution and shows that most of breaking waves were propagating slower than the peak wave speed (mode $=0.39$, mean=0.42). Figure $4-\mathrm{b}$ shows the PDF of the maximum wave breaking length $\left(L_{b}\right)$ normalized by the wavelength computed from the peak wave period using the linear dispersion relation, $L_{p}$. The behaviour of $p\left(L_{b} / L_{p}\right)$ also followed a log-normal PDF for both all breaking waves and dominant waves. This PDF indicates that the majority of the wave breaking events were small whitecaps. Finally, Figure 4-c shows the PDF of the wave breaking period $\left(T_{b}\right)$ normalized by peak wave period $\left(T_{p}\right)$, which also followed a log-normal PDF with mean $=0.11$ and mode $=0.06$. These results are discussed below in Section 4.

Based on the research initially presented in Banner et al. [2] and extended in several other studies [31,32], the number of dominant breaking waves should present a direct correlation with the dominant wave steepness $\left(S_{p}\right.$, calculated as $H_{m 0} / L$ in which $L=$ 
(a)
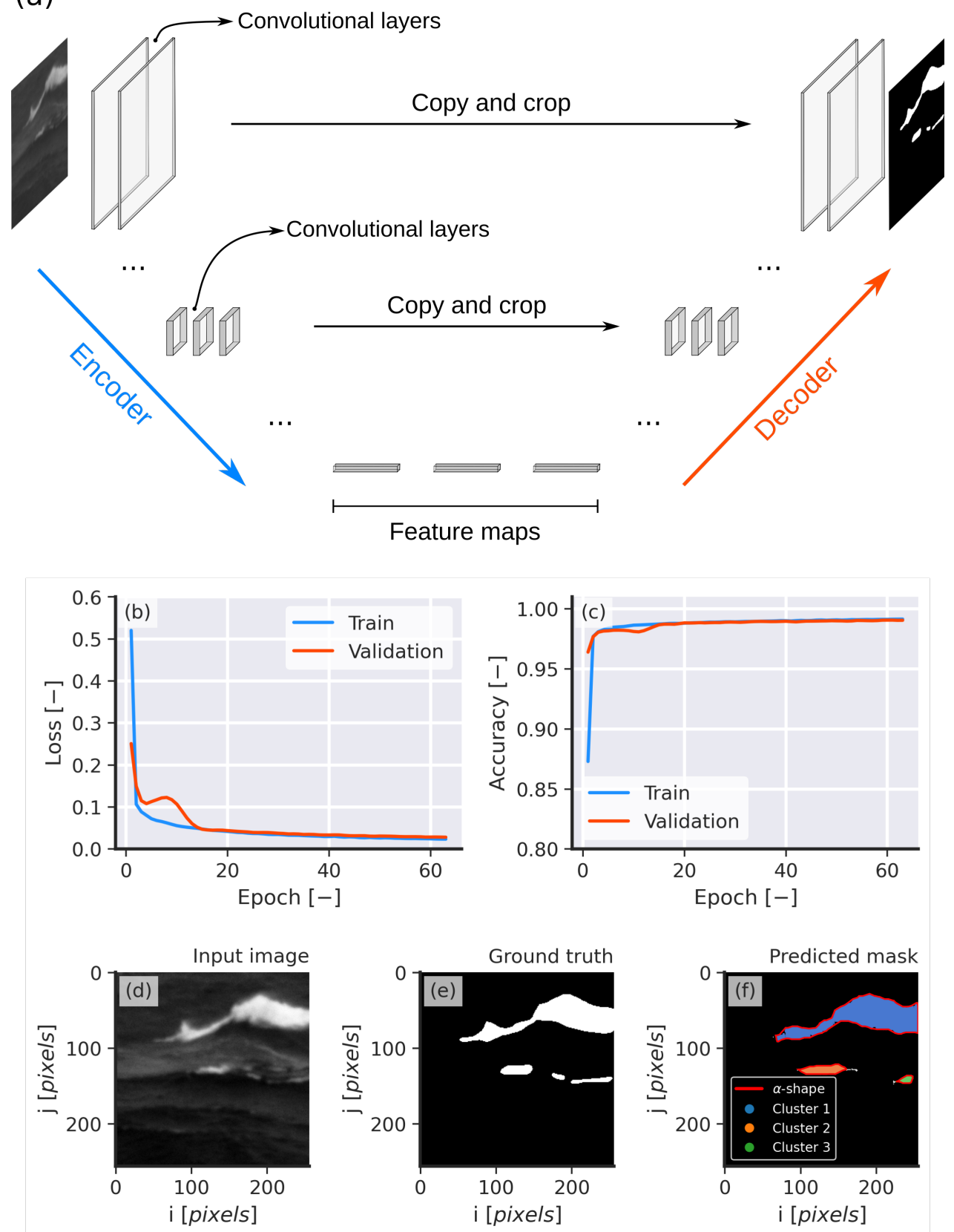

Figure 3. Wave breaking detection method and results. (a) Schematic representation of the U-net Xception model. Note that the layers indicate in this figure are merely for illustration and do not correspond to all layers present in the model, please refer to https: / github.com/caiostringari/ deepwaves/tree/master/segmentation for our formal models. (b) Evolution of the loss function (sparse cross-entropy) for the training and validation datasets. (c) Evolution of the accuracy score for the training and validation datasets. (d) Input test image, (e) Ground truth mask. Predicted mask and clustering of unique foam patches (colors) and their respective contours extracted with $\alpha$-shape (red lines). 
$\left.\frac{g}{2 \pi} T_{p}^{2}\right)$ and, to a lesser extent, with wave age $\left(c_{p} / u_{*}\right)$ and not with wave height $\left(H_{m 0}\right)$. Here, the occurrence rate of waves per unit of area and time per peak wave period is used as a proxy to investigate such correlations. This parameter is calculated as:

$$
R_{t, d, p}=N_{d, t, p} / A / T / T_{p} \quad\left[\text { number of waves } / \mathrm{m}^{2} / \mathrm{s}^{2}\right],
$$

where $N$ is the number of breaking waves indicated, respectively, by the subscripts $t, d$ and $p$ for total, dominant and plunging waves, $A$ is the total analysed area in square meters, $T$ is the total analysis duration in seconds and $T_{p}$ is the wave peak period in seconds. Table 2 shows the values of $R_{d, t, p}$ for all analysed storms. Figure 4 shows the correlations between $R_{d}$ and $H_{m 0}, S_{p}$ and $c_{p} / u_{*}$ for the present data. As expected, no statistically significant correlation $\left(r_{x y}=0.37, p \approx 0.29\right)$ was found between $R_{d}$ and significant wave height $\left(H_{m 0}\right)$, as shown in Figure 4-d. Stronger correlations were found between $R_{d}$ and wave steepness $\left(S_{p}\right)\left(r_{x y}=0.63, p \approx 0.05\right)$ and between $R_{d}$ and wave age $\left(c_{p} / u_{*}\right)\left(r_{x y}=0.57, p \approx 0.08\right)$. Nearly identical results were found if limiting the significant wave height and wave steepness to the same spectral bands as defined by Banner et al. [2]. These results are not shown here because 1) simplicity and 2) practicability as, almost always, engineers only have access to the so-called peak wave parameters (for example, $T_{p}$ ).

Table 2: Wave breaking statistics. Total experiment duration $(T)$, number of wave breaking events $\left(N_{b}\right)$, number of dominant wave breaking events $\left(N_{d}\right)$, ratio between total and dominant events $\left(N_{d} / N_{b}\right)$, number of plunging breakers $\left(N_{p}\right)$ and ratio between all and plunging events $\left(N_{p} / N_{b}\right)$, occurrence rate of breaking waves per area and time normalized by peak wave period for all breaking waves $\left(R_{t}=N_{b} / A / T / T_{p}\right)$, dominant breaking waves $\left(R_{d}=N_{d} / A / T / T_{p}\right)$ and plunging waves $\left(R_{p}=N_{b} / A / T / T_{p}\right)$. For all events, the total area $(A)$ is $8107.9 \mathrm{~m}^{2}$.

\begin{tabular}{llllllllll}
\hline Date & $\begin{array}{l}\mathrm{Nb} \\
{[\mathrm{s}]}\end{array}$ & $\begin{array}{l}N d \\
{[-]}\end{array}$ & $\begin{array}{l}N_{d} / N_{b} \\
{[-]}\end{array}$ & $\begin{array}{l}N p] \\
{[-]}\end{array}$ & $\begin{array}{l}N_{p} / N_{b} \\
{[\%]}\end{array}$ & $\begin{array}{l}R_{t} \\
\text { waves } / m^{2} / s^{2}\end{array}$ & $\begin{array}{l}R_{d} \\
\text { waves } / m^{2} / s^{2}\end{array}$ & $\begin{array}{l}R_{p} \\
\text { waves } / m^{2} / s^{2}\end{array}$ \\
\hline 2019-10-26 09:53:48 & 1196.2 & 698 & 276 & 39.5 & 1 & 0.14 & $6.41 \times 10^{-6}$ & $2.53 \times 10^{-6}$ & $9.18 \times 10^{-9}$ \\
2019-11-02 07:34:01 & 1187.0 & 453 & 129 & 28.5 & 1 & 0.22 & $3.83 \times 10^{-6}$ & $1.10 \times 10^{-6}$ & $8.52 \times 10^{-9}$ \\
2019-11-04 15:04:57 & 1196.8 & 156 & 34 & 21.8 & - & - & $1.25 \times 10^{-6}$ & $2.73 \times 10^{-7}$ & - \\
2019-11-07 15:36:02 & 1191.2 & 538 & 119 & 22.1 & - & - & $4.90 \times 10^{-6}$ & $1.08 \times 10^{-6}$ & - \\
2019-11-12 08:25:27 & 1196.6 & 491 & 36 & 7.3 & 1 & 0.20 & $2.68 \times 10^{-6}$ & $1.97 \times 10^{-7}$ & $5.46 \times 10^{-9}$ \\
2019-11-27 10:00:05 & 1199.2 & 756 & 230 & 30.4 & - & - & $7.39 \times 10^{-6}$ & $2.25 \times 10^{-6}$ & - \\
2019-12-09 09:10:37 & 1199.0 & 284 & 54 & 19.4 & 1 & 0.35 & $1.61 \times 10^{-6}$ & $3.06 \times 10^{-7}$ & $5.66 \times 10^{-9}$ \\
2019-12-13 09:00:05 & 1194.6 & 455 & 102 & 22.4 & 2 & 0.44 & $3.52 \times 10^{-6}$ & $7.90 \times 10^{-7}$ & $1.55 \times 10^{-8}$ \\
2019-12-15 11:42:58 & 1155.2 & 163 & 13 & 8.0 & - & - & $1.13 \times 10^{-6}$ & $9.02 \times 10^{-8}$ & - \\
2019-12-22 11:00:05 & 1198.4 & 689 & 128 & 18.6 & - & - & $4.25 \times 10^{-6}$ & $7.90 \times 10^{-7}$ & - \\
\hline Total & & 4683 & 1121 & 21.7 & 6 & 0.13 & & & \\
\hline
\end{tabular}



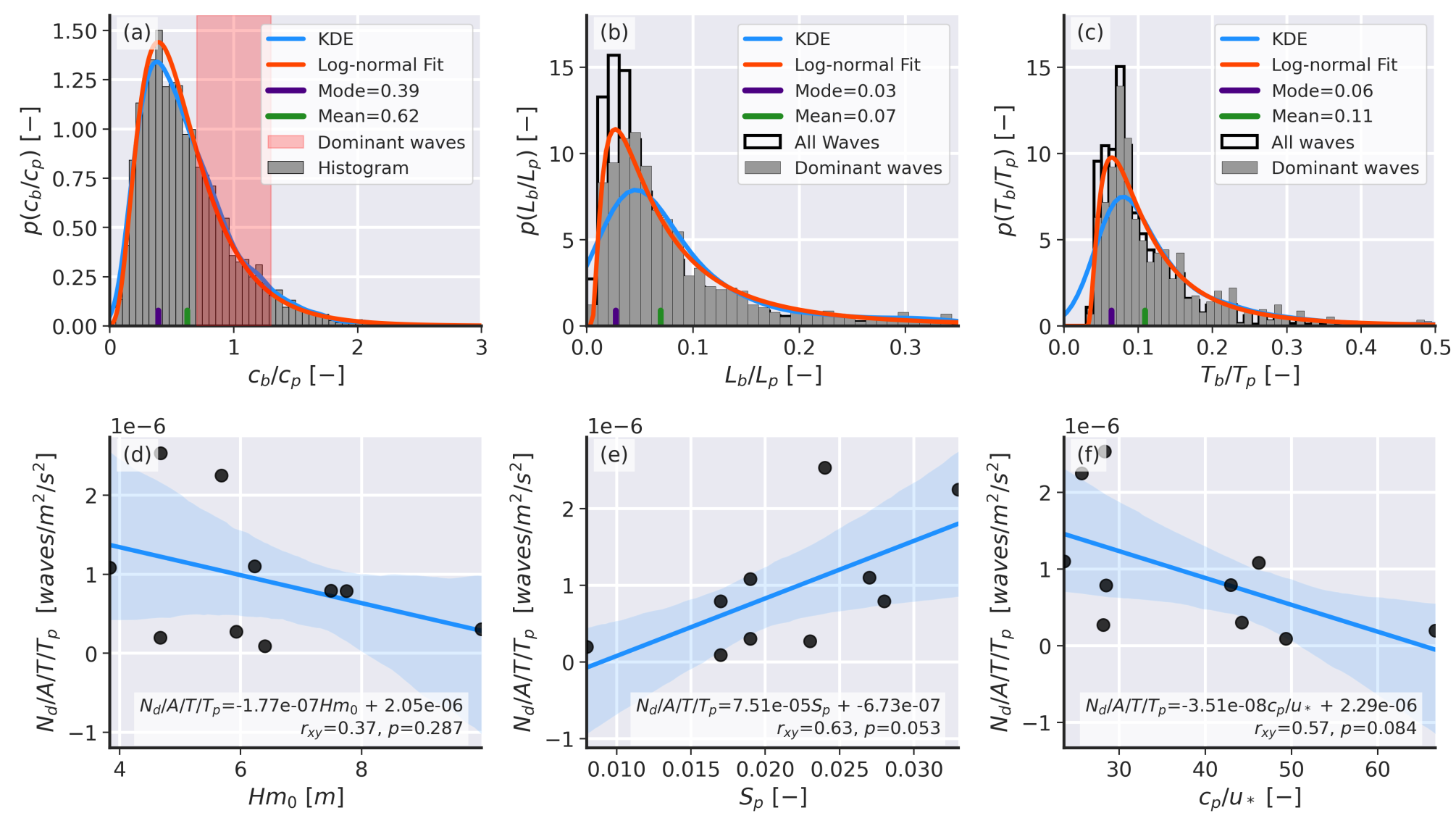

Figure 4. Wave breaking statistics. (a) Probability density function (PDF) of wave breaking speed normalized by peak wave speed $\left(p\left(c_{b} / c_{p}\right)\right)$. The red swash indicates the dominant wave bandwidth. (b) PDF of the wave breaking length normalized by peak wave length $\left(p\left(L_{b} / L_{p}\right)\right)$. (c) PDF of wave breaking duration normalized by peak wave period $\left(p\left(T_{b} / T_{p}\right)\right)$. In (a), (b) and (c) the grey bars show the density histogram, the blue line shows the Kernel Density Estimation (KDE) considering a Gaussian kernel, the orange line shows the log-normal fit to the data, the green tick indicates the mean and the purple tick indicates the mode. In (b) and (c) the filled bars indicate the PDF only for dominant waves where as the open bars show the PDF for all breaking waves. (d) Correlation between $H_{m 0}$ and the occurrence rate of dominant waves per area and time and normalized by peak wave period $\left(R_{d}=N_{d} / A / T / T_{p}\right)$. (e) Correlation between the wave steepness $\left(S_{p}\right)$ and $R_{d}$. (f) Correlation between wave age $\left(c_{p} / u_{*}\right)$ and $\left(R_{d}\right)$. In (d), (e) and (f) the blue swash indicates the $95 \%$ confidence interval for the regression, $r_{x y}$ is Pearson's correlation coefficient and each marker corresponds to a storm from Table 2. 


\subsection{Plunging Waves}

From the results presented above, six waves could be considered intermediate to deep water plunging waves. Out of these six waves, two waves appeared to be particularly severe and were selected for a more detailed analysis. The first wave (Figure 5-a, wave \#1 hereafter) occurred at 26/10/2019 10:11:30. The sea-state waves characterized by $H_{m 0}=4.7 \mathrm{~m}, T_{p}=11.2 \mathrm{~s}$ and south-westerly winds blowing at $15.9 \mathrm{~ms}^{-1}$. This was the only plunging wave observed for this record. The maps in Figure 6 indicate that no opposing currents (Figure 6-a and b) and no opposing synoptic winds (Figure 6-c) were occurring. Local winds, however, may have been opposing given the characteristic foam wisps coming out the wave seen in Figure 5-a. The second wave (Figure 5-b, wave \#2 hereafter) occurred at 13/12/2019 09:08:55 and is clearly larger than the first one. The sea-state was characterized by $H_{m 0}=7.8 \mathrm{~m}, T_{p}=13.3 \mathrm{~s}$ and north-westerly winds blowing at $17.9 \mathrm{~m} / \mathrm{s}$. Opposing currents (Figure 6-d and e) were occurring during this event. The winds were blowing in the same directions as the waves synoptically and locally (note the absence of foam wisps). This wave was visually observed to be the largest wave in its wave group. Interestingly, this particular sea-state produced another plunging wave. Also interestingly, no plunging waves were observed for the most severe storm which had $H_{m 0}$ close to $10 \mathrm{~m}$ which can be linked to the low wave steepness for this storm when compared to the others (See table 1) or could simply be due to the stochastic nature of the wave field combined with the relatively short duration of the records.
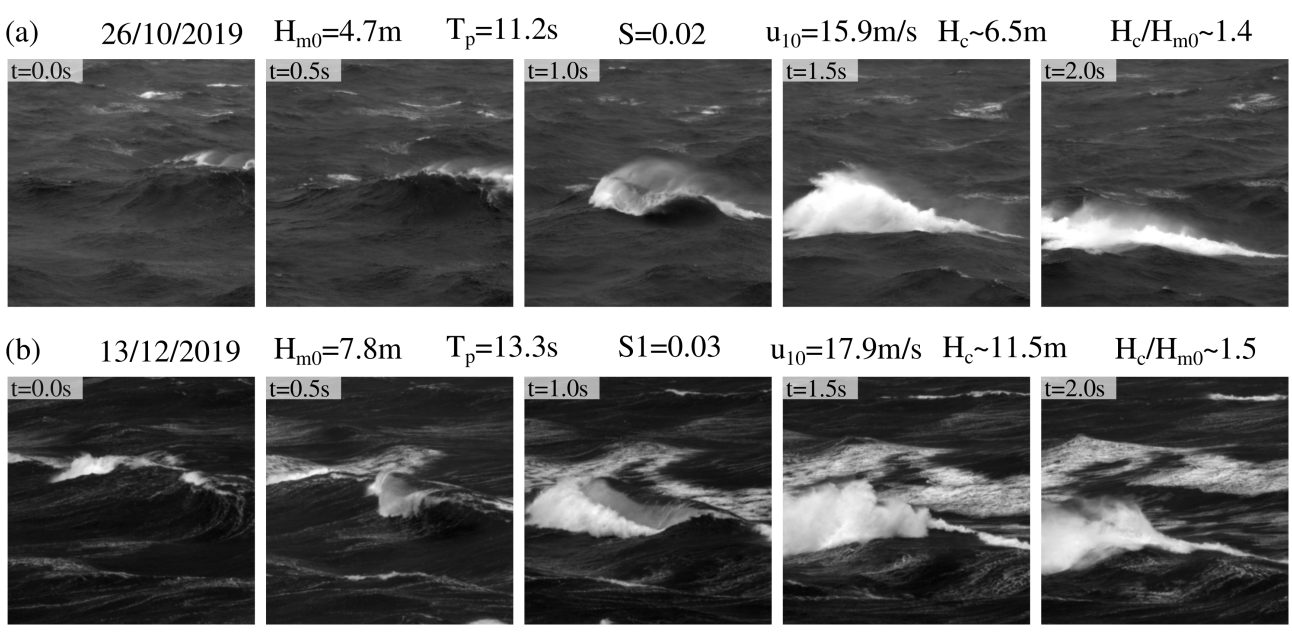

Figure 5. Temporal evolution of two deep water plunging breakers. (a) Wave \#1 recorded at 26/10/2019. (b) Wave \#2 recorded at 13/12/2019. Note the characteristic three-dimensional structure of the waves, the amount of air being trapped and the great amount of white water being generated as the plunging face of the wave collapses into the water. In both cases, the environmental conditions are shown in the figure. In this plot, $H_{c}$ indicates the crest height. 

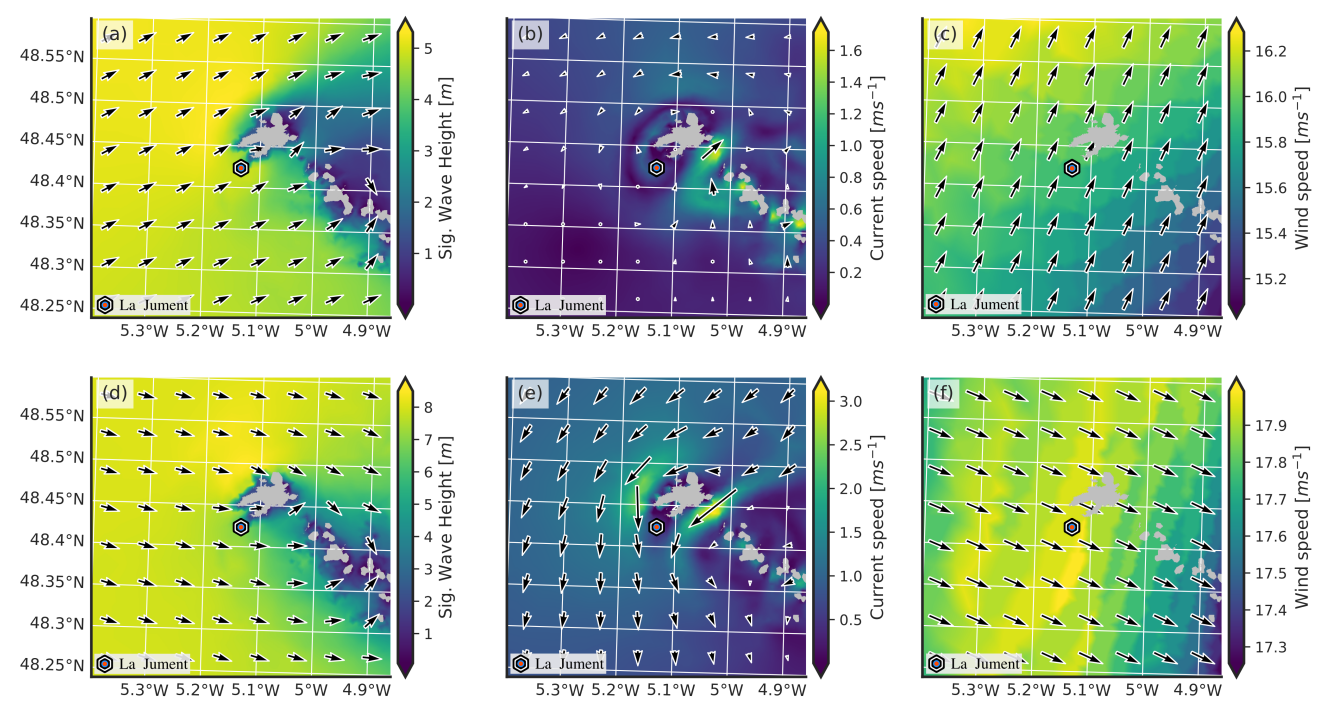

Figure 6. Spatial maps of environmental parameters that help to explain the waves seen in Figure 5. (a) Significant wave height $\left(H_{m 0}\right)$, (b) current speed and direction and (c) wind speed and direction for wave \#1 (26/10/2019 10:11:30). (d) Significant wave height $\left(H_{m 0}\right)$, (e) current speed and direction and (f) wind speed and direction for wave \#2 (13/12/2019 09:08:55). In all plots, data were from the HOMERE and were linearly interpolated from a non-structured grid to a regular grid with $25 \mathrm{~m}$ resolution. Vectors were re-sampled to a coarser resolution to improve visualization.

\section{Discussion}

We have presented a systematic search for intermediate to deep water, dominant breaking waves with particular focus on plunging waves using remote sensing techniques from a lighthouse that is highly exposed to severe sea-states. To our knowledge, this is the first time such search has been attempted using complex field data collected in intermediate to deep waters. The results obtained here make it clear that the recent advances on data-driven research, particularly deep learning, will enable researchers to investigate phenomena of ever-increasing complexity. While the present method is robust enough to separated advected foam from actively breaking waves, it was impossible to distinguish between dominant spilling breakers and small whitecaps that appear on the crest of non-breaking waves (which we assume to be created due to modulation from longer waves), even for a experienced observer. Future research should dedicate more attention to this issue because the phase speed of the short breaking waves (when not modulated by long waves) is much smaller than the speed from the underlying (non-breaking) long wave, which may lead to biases in wave breaking statistics, such as in Phillips' $\Lambda(c) d c$ distribution. Another limitation of the method is that the neural network does not take into account the time evolution of the waves. This means that each frame of the video record is treated as an independent sample (the temporal tracking 
was done a-posteriori with SORT). Future research should focus on developing models that are time-aware, which should eliminate the need for OPTICS and SORT.

The analysis presented in Section 3.1 showed patterns can be at least qualitatively linked to previous studies. For instance, $p\left(c_{b} / c_{p}\right)$ (Figure 4-a) is consistent with field data from Gemmrich et al. [33] (their Figure 3) and indicates that most of breaking waves travel at phase velocity significantly lower than $c_{p}$. This result is consistent with the fact that most of the wave breaking events are small compared to the dominant wavelength (see the $p\left(L_{b} / L_{p}\right)$ distribution in Figure 4-b, for example) and thus are travelling at speeds much slower than $c_{p}$. Another factor that may contribute to the appearance of $p\left(c_{b} / c_{p}\right)$ is the intrinsic slowdown of breaking crests discussed by Fedele et al. [34] that arises due to the non-linear characteristics of ocean waves. Further, $p\left(T_{b} / T_{p}\right)$ (Figure 4-c) is consistent with field data from Gemmrich and Farmer [35] (their Figure 2) but appears to be one order of magnitude smaller than previously reported laboratory data $[4,6,36]$. This difference could be due to the much more controlled laboratory conditions in which the full evolution of wave breaking, including very small scale features, can be precisely quantified. Finally, To the authors' knowledge, the behaviour of $p\left(L_{b} / L_{p}\right)$ has not been yet discussed in the literature. The heavy heavy tail of this distribution, however, appears to hint self-similar behaviour of the breaking wave geometry, at least for the largest observed events. Of course, large uncertainties are present in the data analysed here and the results from Section 3.1 should be interpreted carefully.

The correlations between wave properties and $R_{d}$ also presented the expected behaviour. For instance, the correlation between $R_{d}$ and $S_{p}$ and $R d$ and $c_{p} / u_{*}$ are qualitatively consistent with both the results seen in Banner et al. [2] and with several other observations that correlate wind speeds to wave breaking probabilities (Melville and Matusov [37], for example). For the present data, the direct correlation between $S_{p}$ and $R_{d}$ indicates that steeper dominant waves are more likely to break (Figure 4-d) and younger sea-states generate more dominant breaking waves (Figure 4-f), which can be explained by higher steepness and direct forcing of the wind on the waves. The same correlations were observed between $R_{t}$ and $S_{P}$ and $R_{t}$ and $c_{p} / u_{*}$ but for brevity, these are not discussed here. Due to the small number of plunging waves, a similar analysis for $R_{p}$ would have little statistical meaning and was not attempted here. Future research should focus on correlating the occurrence of plunging waves to similar wave parameters. This will require the development of automated plunging wave detection techniques, a much greater number of sampled storms and longer timeseries, which 
were beyond the scope of this paper. Finally, note that the total number of waves (broken and unbroken) as a function of space and time is unknown in the present study, which precludes the direct calculation of wave breaking probabilities.

One important question still remains: could the two waves from Section 3.2 be considered rogue waves? Due to the issues described in Section 2.1, a reliable stereo video reconstruction was impossible and only approximations of the height of these waves could be achieved. Following the same approach as recently used by Cavaleri et al. [9] (see their Figure 4 and Equation 1), wave \#1 had a approximate crest height $\left(H_{c}\right)$ of $6.5 \mathrm{~m}$ and crest length of $45.1 \mathrm{~m}$ and wave \#2 had a crest height of $11.5 \mathrm{~m}$ and crest length of $145.8 \mathrm{~m}$. Considering the observed sea-state and the usual criterion for rogue wave identification of $1.25 H_{m 0}$, both waves could be considered rogue waves. Interestingly, a wave with $H_{c}=9.5 \mathrm{~m}$ was observed breaking as a spilling breaker less than $100 \mathrm{~m}$ away from wave \#2 at the same time. We highlight, however, that the values reported here are only estimations and are subject to errors. Also note that the the $H_{m 0}$ reported for the first wave is from HOMERE, which indicates that it could be underestimated. This result is, however, in accordance with several rogue wave studies that indicate that the occurrence of these waves are far more likely than previously thought $[38,39]$. Moreover, the observed crest lengths, particularly for wave \#1, indicate that the usual assumption that the breaking crest lateral extension is much larger than the dimension of offshore structures is not always valid. The implications of previous findings about rogue waves and the present observations of plunging waves in intermediate to deep water are important for the design of offshore structures. As mentioned in the Introduction, Goda's formula will predict a much more intense slamming force for these waves than for spilling or non-breaking waves, which should be considered with increased attention by the engineering community in the design of future offshore structures.

Finally, while it is impossible to precisely know the interconnections between waves, winds and currents that led to the occurrence of waves \#1 and \#2, it is possible to search for the most likely causes. From the analysis of Figure 1-d, the directional spreading was low for both waves, which indicates that crossing-seas were not a direct cause. Although the synoptic wind field seems to be aligned with the peak wave direction for both waves, clear foam wisps are seen coming out of wave \#1, which indicates locally opposing wind maybe the likely cause. This wind condition has been shown to enhance wave steepness in the surf zone [13] and may have been a decisive factor creating this wave. While no opposing current was present for wave \#1, a strong opposing current jet is clearly seen 
in Figure 6-e. It is well established that strong opposing currents lead to enhanced wave steepness and rogue waves [12], hence this is the most likely cause of wave \#2. Finally, for both waves, the classic modulational instability cannot be ruled out as cause. The uncertainties as to what factors caused these extreme waves only highlight the need for further research into this subject and facilities such as the ones La Jument lighthouse will surely pave a way forward.

\section{Conclusions}

This paper presented a systematic search for intermediate to deep water, dominant waves with particular focus on plunging, breaking waves during extreme storm conditions. The combination of state-of-the-art remote sensing, data-driven techniques and the real-world laboratory facility available at La Jument lighthouse were key aspects that the made the research presented here possible. The results showed that from all identified wave breaking events $\left(N_{b}=4683\right), 21.7 \%$ represented dominant waves and six waves (or $0.13 \%$ of all breaking waves) could be undoubtedly considered plunging breaking waves. On average, the amount of breaking waves per unit area and time per by peak wave period were in the order of $10^{-6}, 10^{-7}$ and $10^{-9}$, for all breaking waves, dominant breaking waves and plunging waves, respectively. The occurrence rate of dominant waves correlated well with wave steepens and wave age but not with wave height, as expected. In the context of renewable energies and offshore structures, these are important findings, particularly regarding the number of plunging waves, because the slamming force of these waves has a very different nature from their, more frequent, spilling counterparts. Although these events are rare, this work has shown that they do occur and should be accounted for in the design of future offshore structures, particularly the ones that aim to harvest energy in extreme offshore environments, such as floating offshore wind turbines. Unfortunately, several problems occurred during our field experiments which precluded us from obtaining robust quantitative data. It is anticipated, however, that the next data acquisition campaigns at La Jument will fill this gap and provide further quantitative insight into extreme breaking waves. The methods used here are, nonetheless, a robust way to identify and track breaking waves and should provide a solid basis for subsequent field studies.

Author Contributions: Conceptualization, C.E.S and J-F.F..; methodology, C.E.S, J-F.F., F.L. and R.D.; software, C.E.S; validation, C.E.S, F.L. and R.D.; formal analysis, C.E.S.; investigation, C.E.S, J-F.F. and F.L.; resources, J-F.F.; data curation, C.E.S and R.D.; writing-original draft preparation, C.E.S; writing—review and editing, C.E.S, J-F.F., F.L. and R.D. .; visualization, C.E.S.; supervision, 
J-F.F.; project administration, J-F.F.; funding acquisition, J-F.F.. All authors have read and agreed to the published version of the manuscript.

Funding: This work benefited from France Energies Marines and State financing managed by the National Research Agency under the Investments for the Future program bearing the reference numbers ANR-10-IED-0006-14, ANR-10-IEED-0006-26, ANR-10-IEED-0006-20 for the projects DiME, CARAVELE and DIMPACT.

Institutional Review Board Statement: Not applicable.

Informed Consent Statement: Not applicable.

Data Availability Statement: Code and neural networks used here are available from Zenodo [40]. The data necessary to reproduce the results seen in this paper are also available from Zenodo [41].

Acknowledgments: We would like to thank the Service des Phares et Balises for providing crucial support in reaching La Jument lighthouse and maintaining energy generators for our computer and cameras and the Service hydrographique et océanographique de la Marine (Shom) for the support in deploying the Datawell wave buoy, processing raw buoy data and sharing their data.

Conflicts of Interest: The authors declare no known conflict of interest.

\section{References}

1. Longuet-Higgins, M.S. Mechanisms of Wave Breaking in Deep Water. In Sea Surface Sound; Kerman, B.R., Ed.; Springer, Dordrecht, 1988; pp. 1-30. doi:https://doi.org/10.1007/978-94-009-3017-9_1.

2. Banner, M.L.; Babanin, A.V.; Young, I.R. Breaking Probability for Dominant Waves on the Sea Surface. Journal of Physical Oceanography 2000, 30, 3145-3160. doi:10.1175/1520-0485(2000)030<3145:BPFDWO>2.0.CO;2.

3. Peureux, C.; Ardhuin, F.; Guimarães, P.V. On the Unsteady Steepening of Short Gravity Waves Near the Crests of Longer Waves in the Absence of Generation or Dissipation Journal of Geophysical Research : Oceans. Journal of Geophysical Research: Oceans 2020, 126, 1-16. doi:10.1029/2020JC016735.

4. Rapp, R.J.; Melville, W.K. Laboratory measurements of deep-water breaking waves. Philosophical Transactions of the Royal Society A: Mathematical, Physical and Engineering Sciences 1990, 331, 735-808.

5. McAllister, M.L.; Draycott, S.; Adcock, T.A.; Taylor, P.H.; Van Den Bremer, T.S. Laboratory recreation of the Draupner wave and the role of breaking in crossing seas. Journal of Fluid Mechanics 2018, 860, 767-786. doi:10.1017/jfm.2018.886.

6. Craciunescu, C.C.; Christou, M. Wave breaking energy dissipation in long-crested focused wave groups based on JONSWAP spectra. Applied Ocean Research 2020, 99, 102144. doi:https:/ / doi.org/10.1016/j.apor.2020.102144.

7. Banner, M.L.; Peregrine, D.H. Wave Breaking in Deep Water. Annual Review of Fluid Mechanics 1993, $25,373-397$. doi:https://doi.org/10.1146/annurev.fl.25.010193.002105.

8. Donelan, M.A.; Magnusson, A.K. The Making of the Andrea Wave and other Rogues. Scientific Reports $2017,7,1-7$. doi:10.1038/srep44124. 
9. Cavaleri, L.; Barbariol, F.; Bastianini, M.; Benetazzo, A.; Bertotti, L.; Pomaro, A. An exceptionally high wave at the CNR-ISMAR oceanographic tower in the Northern Adriatic Sea. Scientific Data 2021, 8, 1-7. doi:10.1038/s41597-021-00825-x.

10. Adcock, T.A.A.; Taylor, P.H. The physics of anomalous ('rogue') ocean waves. Reports on Progress in Physics $2014,77,105901$. doi:10.1088/0034-4885/77/10/105901.

11. Fedele, F.; Brennan, J.; Ponce De León, S.; Dudley, J.; Dias, F. Real world ocean rogue waves explained without the modulational instability. Scientific Reports 2016, 6, 1-11. doi:10.1038/srep27715.

12. Lu, W.; Yang, J.; Li, X. Evolution of the Rogue Waves under the Influence of the Wave-Current Interference , 2016.

13. Sous, D.; Forsberg, P.L.; Touboul, J.; Gonçalves Nogueira, G. Laboratory experiments of surf zone dynamics under on- and offshore wind conditions. Coastal Engineering 2021, 163, 103797. doi:10.1016/j.coastaleng.2020.103797.

14. Filipot, J.F.; Guimaraes, P.; Leckler, F.; Hortsmann, J.; Carrasco, R.; Leroy, E.; Fady, N.; Accensi, M.; Prevosto, M.; Duarte, R.; Roeber, V.; Benetazzo, A.; Raoult, C.; Franzetti, M.; Varing, A.; Le Dantec, N. La Jument lighthouse: a real-scale laboratory for the study of giant waves and their loading on marine structures. Philosophical Transactions of the Royal Society A: Mathematical, Physical and Engineering Sciences 2019, 377, 20190008. doi:10.1098/rsta.2019.0008.

15. Chella, M.A.; Tørum, A.; Myrhaug, D. An overview of wave impact forces on offshore wind turbine substructures. Energy Procedia 2012, 20, 217-226. doi:10.1016/j.egypro.2012.03.022.

16. Goda, Y. A study on impulsive breaking wave force upon a vertical pile. Rept. Port and Harbour Res. Inst. 1966, 5, 1-30.

17. Boudière, E.; Maisondieu, C.; Ardhuin, F.; Accensi, M.; Pineau-Guillou, L.; Lepesqueur, J. A suitable metocean hindcast database for the design of Marine energy converters. International Journal of Marine Energy 2013, 3-4, 40-52. doi:10.1016/j.ijome.2013.11.010.

18. Accensi, M.; Maisondieu, C. HOMERE, 2015. doi:10.12770/cf47e08d-1455-4254-955e-d66225c9dc90.

19. Hersbach, H.; Bell, B.; Berrisford, P.; Hirahara, S.; Horányi, A.; Muñoz-Sabater, J.; Nicolas, J.; Peubey, C.; Radu, R.; Schepers, D.; Simmons, A.; Soci, C.; Abdalla, S.; Abellan, X.; Balsamo, G.; Bechtold, P.; Biavati, G.; Bidlot, J.; Bonavita, M.; De Chiara, G.; Dahlgren, P.; Dee, D.; Diamantakis, M.; Dragani, R.; Flemming, J.; Forbes, R.; Fuentes, M.; Geer, A.; Haimberger, L.; Healy, S.; Hogan, R.J.; Hólm, E.; Janisková, M.; Keeley, S.; Laloyaux, P.; Lopez, P.; Lupu, C.; Radnoti, G.; de Rosnay, P.; Rozum, I.; Vamborg, F.; Villaume, S.; Thépaut, J.N. The ERA5 global reanalysis. Quarterly Journal of the Royal Meteorological Society 2020, 146, 1999-2049. doi:10.1002/qj.3803.

20. Coles, S. An introduction to extreme values, 3rd ed ed.; Springer-Verlag London 2001: London, 2015; p. 219. doi:10.1007/978-1-44713675-0.

21. Holman, R.A.; Stanley, J. The history and technical capabilities of Argus. Coastal Engineering 2007, 54, 477-491. doi:10.1016/j.coastaleng.2007.01.003.

22. Stringari, C.E.; Veras Guimarães, P.; Filipot, J.F.; Leckler, F.; Duarte, R. Deep neural networks for active wave breaking classification. Scientific Reports 2021, 11, 3604. doi:10.1038/s41598-021-83188-y.

23. Chollet, F. Xception: Deep learning with depthwise separable convolutions. Proceedings - 30th IEEE Conference on Computer Vision and Pattern Recognition, CVPR 2017 2017, 2017-January, 1800-1807, [1610.02357]. doi:10.1109/CVPR.2017.195.

24. Ronneberger, O.; Fischer, P.; Brox, T. U-net: Convolutional networks for biomedical image segmentation. Medical Image Computing and Computer-Assisted Intervention - MICCAI 2015. MICCAI 2015. Lecture Notes in Computer Science; Navab, N.; Hornegger, J.; Wells, W.; Frangi, A., Eds. Springer, Cham, 2015, Vol. 9351, pp. 234-241, [1505.04597]. doi:10.1007/978-3-319-245744_28. 
25. Tan, C.; Sun, F.; Kong, T.; Zhang, W.; Yang, C.; Liu, C. A Survey on Deep Transfer Learning. Artificial Neural Networks and Machine Learning - ICANN 2018; Krurkova, V.; Manolopoulos, Y.; Hammer, B.; Iliadis, L.; Maglogiannis, I., Eds.; Springer International Publishing: Cham, 2018; pp. 270-279.

26. Ankerst, M.; Breunig, M.M.; Kriegel, H.p. OPTICS : Ordering Points To Identify the Clustering Structure. ACM SIGMOD’99 Int. Conf. on Management of Data; , 1999; pp. 49-60.

27. Bewley, A.; Ge, Z.; Ott, L.; Ramos, F.; Upcroft, B. Simple online and realtime tracking. Proceedings - International Conference on Image Processing, ICIP 2016, 2016-Augus, 3464-3468, [1602.00763]. doi:10.1109/ICIP.2016.7533003.

28. Edelsbrunner, H.; Kirkpatrick, D.G.; Seidel, R. On the Shape of a Set of Points in the Plane. IEEE Transactions on Information Theory 1983, 29, 551-559. doi:10.1109/TIT.1983.1056714.

29. Phillips, O.M. Spectral and statistical properties of the equilibrium range in wind-generated gravity waves. Journal of Fluid Mechanics 1985, 156, 505-531. doi:10.1017/S0022112085002221.

30. Grilli, S.T.; Svendsen, I.A.; Subramanya, R. Breaking Criterion and Characteristics for Solitary Waves on Slopes. Journal of Waterway, Port, Coastal, and Ocean Engineering 1997, 123, 102-112. doi:10.1061/(asce)0733-950x(1997)123:3(102).

31. Banner, M.L.; Gemmrich, J.R.; Farmer, D.M. Multiscale measurements of ocean wave breaking probability. Journal of Physical Oceanography 2002, 32, 3364-3375. doi:10.1175/1520-0485(2002)032<3364:MMOOWB>2.0.CO;2.

32. Banner, M.L.; Morison, R.P. Refined source terms in wind wave models with explicit wave breaking prediction. Part I: Model framework and validation against field data. Ocean Modelling 2010, 33, 177-189. doi:10.1016/j.ocemod.2010.01.002.

33. Gemmrich, J.R.; Banner, M.L.; Garrett, C. Spectrally resolved energy dissipation rate and momentum flux of breaking waves. Journal of Physical Oceanography 2008, 38, 1296-1312. doi:10.1175/2007JPO3762.1.

34. Fedele, F.; Banner, M.L.; Barthelemy, X. Crest speeds of unsteady surface water waves. Journal of Fluid Mechanics 2020, 899, A5. doi:10.1017/jfm.2020.424.

35. Gemmrich, J.R.; Farmer, D.M. Observations of the scale and occurrence of breaking surface waves. Journal of Physical Oceanography 1999, 29, 2595-2606. doi:10.1175/1520-0485(1999)029<2595:OOTSAO>2.0.CO;2.

36. Perlin, M.; Choi, W.; Tian, Z. Breaking Waves in Deep and Intermediate Waters. Annual Review of Fluid Mechanics 2013, 45, 115-145. doi:10.1146/annurev-fluid-011212-140721.

37. Melville, W.K.; Matusov, P. Distribution of breaking waves at the ocean surface. Nature 2002, 417, 58-63. doi:10.1038/417058a.

38. Fedele, F. Space-time extremes in short-crested storm seas. Journal of Physical Oceanography 2012, 42, 1601-1615. doi:10.1175/JPOD-11-0179.1.

39. Benetazzo, A.; Barbariol, F.; Bergamasco, F.; Torsello, A.; Carniel, S.; Sclavo, M. Observation of extreme sea waves in a space-time ensemble. Journal of Physical Oceanography 2015, 45, 2261-2275. doi:10.1175/JPO-D-15-0017.1.

40. Stringari, C.E. caiostringari/deepwaves: v1, 2021. doi:10.5281/zenodo.4495600.

41. Stringari, C.E.; Leckler, F.; Duarte, R.; Filipot, J.F. A Dataset of Breaking Waves Remotly Sensed at La Jument Lighthouse During the 2019-2020 Winter Storm Season, 2021. doi:10.5281/zenodo.4597088. 